\title{
Current trend for the accurate determination of active pharmaceutical ingredients in blood, serum and urine samples by Mcnts@MIP using chromatographic techniques
}

\begin{abstract}
Magnetic carbon nanotubes with magnetic imprinted polymer (MCNTs@MIP) extraction techniques are great current interest for the determination of active pharmaceutical ingredients. Magnetic carbon nanotubes (MCNTs) can be manipulated by magnetic fields, so that when functionalized, they can be used for the purification and separation of biomolecules and even whole cells. The aim of this study was to understand the interaction between carbon nanotubes (CNTs) and active pharmaceutical ingredients and evaluate the drug-loading ability of CNTs. Molecularly imprinted polymers (MIPs) are artificially synthesized polymeric materials, each of which contains a large number of cavities that are complementary in terms of shape, size, and functional groups present to a specific target molecule. Therefore, MIPs show the ability to recognize specific molecules, and high binding affinities for these target molecules. In recent years, MIPs have been widely used in various fields, such as separations, sensors, and catalysis.
\end{abstract}

Volume I Issue 3 - 2017

\author{
Thippani Ramesh, Pothuraju Nageswara Rao \\ Department of Chemistry, National Institute of Technology \\ Warangal, India
}

Correspondence: Thippani Ramesh, Department of Chemistry, National Institute of Technology Warangal, India, Tel 086832 4436, Email tippaniramesh. I@gmail.com

Received: August 24, 2017 | Published: October 04, 2017
Abbreviations: MIPs, molecularly imprinted polymers; MCNTs@MIP, magnetic carbon nanotubes with magnetic imprinted polymer

\section{Introduction}

The drug discovery and development process has undergone dramatic changes particularly in the last decade. Progress in drug discovery has been fuelled by improvements in methodologies and technologies including automated high performance liquid chromatography (HPLC), LC-MS/MS, GC-MS/MS LC-NMR and high throughput purification methods. ${ }^{1-3}$ At each phase of drug development the analyses of varieties of samples are performed to adequately control and monitor the quality of the prospective drug candidates, excipients, and final products, which require bioanalytical as well as impurity profiling methods. Effective and fast method development is of paramount importance throughout the drug development life cycle. In view of the continuous demand for bioanalytical methods in drug discovery and impurity profiling method for maintaining the quality and safety of drugs, their analysis represents several problems and poses many challenges to analytical chemists. For instance, the administration of drug dosage to the animals is small. It leads to decrease in concentration levels of drug and its metabolites in biological samples like blood, serum and urine etc. Thus determination of trace level drugs in such complex biological fluids requires efficient sample preparation procedures prior to their separation, detection and quantification. ${ }^{4}$

Today, the analytical methodology is in a period of transition due to technological advances and modified policies of the governmental regulatory agencies. In view of above strategy current analysts and researchers are following Magnetic Carbon Nano Tubes with Magnetic Imprinted Polymer (MCNTs@MIP) extraction techniques. Magnetic separation technology has received considerable attention in recent years for its great potential application in cell separation, drug delivery and enzyme immobilization. It can provide the easy removal of template, high adsorption capacity, low mass transportation resistance, and fast adsorption kinetics by applying appropriate magnetic field. The chemical, mechanical and thermal stability together with high selectivity for template molecules, molecularly imprinted polymers (MIPs) have been utilized for a wide variety of applications, including chromatography, ${ }^{5}$ protein separation, ${ }^{6}$ solid-phase extraction, ${ }^{7}$ drugcontrolled release ${ }^{8}$ and sensor devices. ${ }^{9,10}$ The imprinting technique is a well established and simple technique for synthesizing materials with specific molecular recognition properties. Although the bulk MIP prepared by conventional methods exhibits high selectivity, ${ }^{11}$ some disadvantages were suffered, such as the heterogeneous distribution of the binding sites, embedding of most binding sites, and poor site accessibility for template molecule. ${ }^{12}$

To resolve these problems, scientists have made efforts to prepare core-shell structural MIP. By preparing the MIP film on a solid-support substrate, the surface-imprinting technique provides an alternative way to improve mass transfer and reduce permanent entrapment of the template. In previous investigations, $\mathrm{SiO}_{2},{ }^{13} \mathrm{TiO}_{2}{ }^{14}$ and $\mathrm{Fe}_{3} \mathrm{O}_{4}{ }^{15}$ have been widely used in the surface imprinting process. Recently, in order to avoid leakages and fragility of traditional support materials, special attentions have been directed to combine magnetic nano particles (MNPs) with nano sized materials such as carbon nano tubes (CNTs) for magnetic carbon nano tubes (MCNTs) preparation (Figure 1). Various MCNT synthetic methods have been described in the literatures. These nano materials including molecularly imprinted polymers (MIPs), M-MIPs, carbon nano tube (CNTs), magnetic-CNTs (M-CNTs) and magnetic-MIP-CNTs can absorb drugs and other bio molecules by forming stable covalent or non-covalent bonds between nano materials and analyte. ${ }^{16}$ Among these nano materials, magnetic- 
MIP-CNTs seem to be the best candidate not only for drug delivery system in therapeutics but also for SPE in drug analysis. Magnetic molecularly imprinted polymer is a spherical polymer endowed with magnetic properties. ${ }^{17}$ They have highly selective binding characteristics to the target template and homologues. The proposal of preparation of MCNTs by a simple solved thermal process, which can easily alter the size (100-350 nm), location and denseness of $\mathrm{Fe}_{3} \mathrm{O}_{4}$ beads, fixed on CNTs as well as the MCNT structure via controlling the reaction parameters. ${ }^{18}$

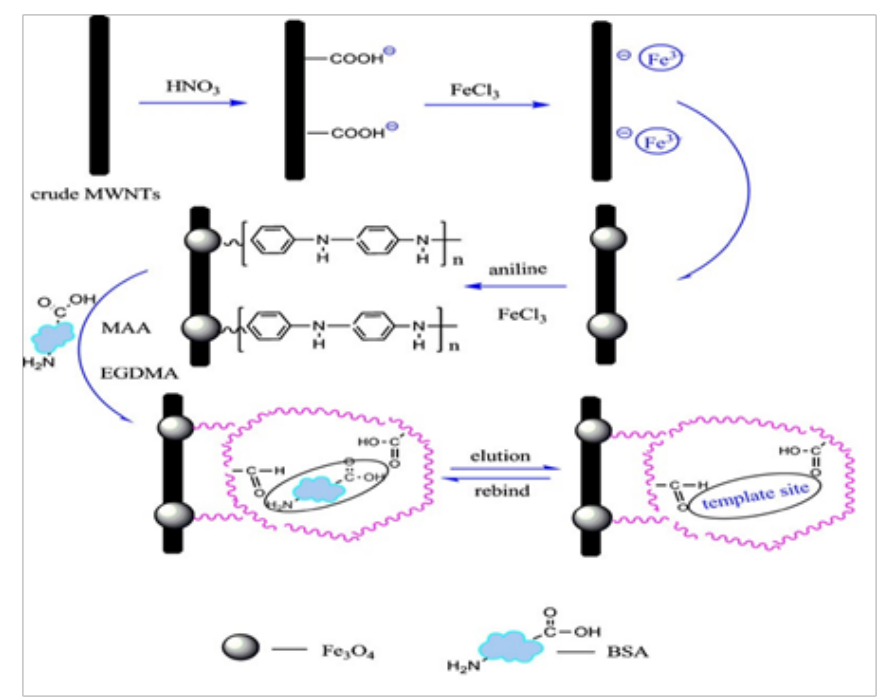

Figure I Procedure for preparing magnetic protein imprinted polymers.

\section{Work flow}

a. Synthesis of MCNTs (magnetic carbon nano tubes), synthesis of MCNTs@MIP (molecularly imprinted polymer based on magnetic carbon nano tubes), study of the capacity of the MIP, binding experiments, adsorption selectivity, characterization, binding characteristics of imprinted materials, applications to biological materials like rat serum sample analysis for some new API drugs and its metabolites using liquid chromatographic analysis.

b. Development of bio analytical method for the determination and quantification of API in adsorbed MCNTs@MIP by liquid chromatography.

c. Finally to provide a more sensitive, selective, rapid and versatile method for the new API molecules than other analytical methods.

\section{Acknowledgements}

None.

\section{Conflict of interest}

The authors declare that there is no conflict of interest.

\section{References}

1. $\mathrm{R}$ Henderson. Managing innovation in the information age. Harvard Business Review. 1994.

2. HH Sedlacek, AA Sapienza, A Volker. Ways to successful strategies in drug research and development. VCH, Weinheim and New York, USA; 1996.
3. Tsinopoulos $\mathrm{C}$, Mccarthy I. The evolution of strategies for drug discovery: From accidental discovery to rational drug design, Proceedings of $R \& D$ management conference. Dublin, Ireland; 2001.

4. Gorog S, Babjak M, Balogh G, et al. Drug impurity profiling strategies. Talanta. 1997;44(9):1517-1526.

5. Zhang Y, Li YW, Hu YL, et al. Preparation of magnetic indole-3-acetic acid imprinted polymer beads with 4 -vinylpyridine and $\beta$-cyclodextrin as binary monomer via microwave heating initiated polymerization and their application to trace analysis of auxins in plant tissues. J Chromatogr A. 2010;1217(47):7337-7344.

6. Zhang $Z$, Yang $X$, Chen $X$, et al. Novel magnetic bovine serum albumin imprinted polymers with a matrix of carbon nanotubes, and their application to protein separation. Anal Bioanal Chem. 2011;401(9):2855-2865.

7. Zhang HB, Zhang ZH, Hu YF, et al. Synthesis of a Novel Composite Imprinted Material Based on Multiwalled Carbon Nanotubes as a Selective Melamine Absorbent. J Agric Food Chem. 2011;59(4):1063.

8. Francesco Puoci, Francesca Iemma, Giuseppe Cirillo, et al. New restricted access materials combined to molecularly imprinted polymers for selective recognition/release in water media. Eur Polym J. 2009;45(6):1634-1640.

9. Chen HJ, Zhang ZH, Luo LJ, et al. Surface-imprinted chitosan-coated magnetic nanoparticles modified multi-walled carbon nanotubes biosensor for detection of bovine serum albumin. Sens Actuators B Chem. 2012;163(1):76-83.

10. Rezaei B, Majidi N, Ensafi AA, et al. Molecularly imprinted-multiwall carbon nanotube paste electrode as a biosensor for voltammetric detection of rutin. Anal Methods. 2011;3(11):2510-2516.

11. Akiyama T, Hishiya T, Asanuma H, et al. Molecular Imprinting of Cyclodextrin on Silica-Gel Support for the Stationary Phase of HighPerformance-Liquid-Chromatography. $J$ Incl Phenom Macrocyclic Chem. 2001;41(1-4):149-153.

12. Kan XW, Zhao Y, Geng ZR, et al. Composites of Multiwalled Carbon Nanotubes and Molecularly Imprinted Polymers for Dopamine Recognition. J Phys Chem C. 2008;112(16):4849-4854.

13. Ramesh T, Nageswara Rao P, Nageswara Rao R. LC-MS/MS characterization of forced degradation products of zofenopril. J Pharm Biomed Anal. 2014;88:609-616.

14. Shen $X$, Zhu L, Huang C, et al. Inorganic molecular imprinted titanium dioxide photocatalyst: synthesis, characterization and its application for efficient and selective degradation of phthalate esters. J Mater Chem. 2009;199(27):4843-4851.

15. Qu P, Lei J, Zhang L, et al. Molecularly imprinted magnetic nanoparticles as tunable stationary phase located in microfluidic channel for enantioseparation. J Chromatogr. A 2010;1217(39):6115-6121.

16. Correa Duarte MA, Grzelczak M, Salgueiriño Maceira V, et al. Alignment of carbon nanotubes under low magnetic fields through attachment of magnetic nanoparticles. J Phys Chem B. 2005;109(41):19060-19063.

17. Gao C, Li W, Morimoto $\mathrm{H}$, et al. Magnetic Carbon Nanotubes: Synthesis by Electrostatic Self-Assembly Approach and Application in Biomanipulations. J Phys Chem B. 2006;110(14):7213-7220.

18. Xiao D, Dramou $\mathrm{P}, \mathrm{He} \mathrm{H}$, et al. Magnetic carbon nanotubes: synthesis by a simple solvothermal process and application in magnetic targeted drug delivery system. J Nanopart Res. 2012;14(11):984. 